\title{
THE CLASS OF A HURWITZ DIVISOR ON THE MODULI OF CURVES OF EVEN GENUS*
}

\author{
GERARD VAN DER GEER ${ }^{\dagger}$ AND ALEXIS KOUVIDAKIS ${ }^{\ddagger}$
}

\begin{abstract}
We study the geometry of the natural map from the Hurwitz space $\bar{H}_{2 k, k+1}$ to the moduli space $\overline{\mathcal{M}}_{2 k}$. We calculate the cycle class of the Hurwitz divisor $D_{2}$ on $\overline{\mathcal{M}}_{g}$ for $g=2 k$ given by the degree $k+1$ covers of $\mathbb{P}^{1}$ with simple ramification points, two of which lie in the same fibre. This has applications to bounds on the slope of the moving cone of $\overline{\mathcal{M}}_{g}$, the calculation of other divisor classes and motivated an algebraic proof for the formula of the Hodge bundle of the Hurwitz space, cf. [12, 11].
\end{abstract}

Key words. Hurwitz space, Hurwitz divisor, moduli of curves.

AMS subject classifications. $14 \mathrm{H} 10,14 \mathrm{H} 51$.

1. Introduction. In this paper we study the geometry of the natural map $\bar{H}_{g, d} \rightarrow \overline{\mathcal{M}}_{g}$ of a Hurwitz space to the moduli space of curves in the case of BrillNoether number zero. We will determine the class of a key divisor (Hurwitz cycle) whose class was still unknown. It can be used to deduce results on the slope of the moving cone of $\overline{\mathcal{M}}_{g}$ and via its connection with the Hodge bundle of the Hurwitz space led to an algebraic proof for the formula of the Hodge bundle (cf. [11, 12]).

Hurwitz cycles are playing a significant role in the study of the geometry of the moduli space $\mathcal{M}_{g}$ of curves of genus $g$. For example, they appeared prominently in the work [16] of Harris and Mumford on the Kodaira dimension of $\mathcal{M}_{g}$. Faber and Pandharipande showed in [7] that the cycle classes of Hurwitz loci are tautological. For some Hurwitz loci the cycles classes are known, but for many such loci the cycle classes are still unknown; for work in this direction see [9].

We work over the complex numbers. The generic curve of even genus $g=2 k$ is in finitely many ways a degree $k+1$ cover of the projective line with $6 k$ branch points. By the Hurwitz-Zeuthen formula these are all simple branch points. Here simple branch point means that the corresponding fiber has exactly one simple ramification point. The condition that two of the resulting $6 k$ ramification points lie in the same fibre over the projective line defines a divisor $D_{2}$ in $\mathcal{M}_{g}$. More precisely, a smooth curve $C$ of genus $g=2 k$ defines a point of $D_{2}$ if it admits a degree $k+1$ map to $\mathbb{P}^{1}$ with simple ramification points and $6 k-1$ branch points. Similarly, the condition that two ramification points collide (and then define a triple ramification point) defines a divisor $D_{3}$ in $\mathcal{M}_{g}$. Their closures give divisors in $\overline{\mathcal{M}}_{g}$, again denoted by $D_{2}$ and $D_{3}$. These divisors are important and appeared already in the paper [14] of Harris.

Harris calculated the class of $D_{3}$ in [14] in 1984, but the class of $D_{2}$ escaped determination so far. By using a recent result of Kokotov, Korotkin and Zograf [18] on the shape of the Hodge bundle on Hurwitz spaces we are now able to calculate this class. In fact, this connection inspired an algebraic proof for this formula, cf. [11].

In order to formulate the result we recall that the Picard group with rational coefficients of the Deligne-Mumford stack $\overline{\mathcal{M}}_{g}$ is generated by the class $\lambda$ of the Hodge bundle and the classes $\delta_{j}$ of the boundary divisors $\Delta_{j}$ for $j=1, \ldots,[g / 2]$.

\footnotetext{
*Received December 14, 2011; accepted for publication February 14, 2012.

${ }^{\dagger}$ Korteweg-de Vries Instituut, Universiteit van Amsterdam, Postbus 94248, 1090 GE Amsterdam, The Netherlands (G.B.M.vanderGeer@uva.nl).

$\ddagger$ Department of Mathematics, University of Crete, GR-71409 Heraklion, Greece (kouvid@ math.uoc.gr).
} 
Our result reads as follows.

THEOREM 1.1. Let $g=2 k$ be an even natural number. The class of $D_{2}$ on $\overline{\mathcal{M}}_{g}$ can be written as $c_{\lambda} \lambda+\sum_{j=0}^{k} c_{j} \delta_{j}$ with the coefficients $c_{\lambda}$ and $c_{j}$ given by

$$
c_{\lambda}=6 N \frac{6 k-1}{2 k-1}(k-2)(k+3),
$$

and

$$
c_{0}=-\frac{2 N}{2 k-1}(k-2)\left(3 k^{2}+4 k-1\right)
$$

and for $1 \leq j \leq k$

$$
c_{j}=-3 N \frac{j(2 k-j)}{2 k-1}\left(6 k^{2}-4 k-7\right)+\frac{9}{2} j(2 k-j) \alpha(k, j) .
$$

Here $N=\left(\begin{array}{c}2 k \\ k+1\end{array}\right) / k$ and $\alpha(k, j)$ is the combinatorial expression

$$
\alpha(k, j)=\frac{j(2 k-j)+k}{k(k+1)}\left(\begin{array}{c}
j \\
{[j / 2]}
\end{array}\right)\left(\begin{array}{c}
2 k-j \\
k-[j / 2]
\end{array}\right) \quad \text { for } j \text { even }
$$

and

$$
\alpha(k, j)=\frac{(j+1)(2 k-j)}{k(k+1)}\left(\begin{array}{c}
j+1 \\
1+[j / 2]
\end{array}\right)\left(\begin{array}{c}
2 k-j-1 \\
k-1-[j / 2]
\end{array}\right) \quad \text { for } j \text { odd }
$$

We determine which boundary divisors of $\bar{H}_{g, d}$ are mapped dominantly to a boundary divisor of $\overline{\mathcal{M}}_{g}$ and those that map dominantly to a divisor of $\mathcal{M}_{g}$. We also prove irreducibility results for the divisors involved and determine the degree of the corresponding maps.

For applications of this to bounding the slope of the movable cone of $\overline{\mathcal{M}}_{g}$ and the calculation of other divisor classes, like the Eisenbud-Harris divisor on $\overline{\mathcal{M}}_{g}$, we refer to [12]. For the determination of the class of the Hodge bundle on Hurwitz spaces we refer to $[11]$.

2. The Hurwitz scheme. We call a degree $d$ cover $C_{1} \rightarrow C_{2}$ of Riemann surfaces simple if every fibre has at least $d-1$ distinct points. Let $H_{g, d}$ be the Hurwitz scheme of simple covers of the projective line $\mathbb{P}^{1}$ of degree $d$ and genus $g$ with ordered branch points and $\bar{H}_{g, d}$ the compactification of the Hurwitz scheme by the admissible covers with an ordering of the branch points, see [16], p. 57. This is an irreducible projective scheme. Recall that two admissible covers $f_{i}: C_{i} \rightarrow P_{i}$ are considered equivalent if there exist isomorphisms $h: C_{1} \rightarrow C_{2}$ and $\gamma: P_{1} \rightarrow P_{2}$ (preserving the markings) with $f_{2} \circ h=\gamma \circ f_{1}$.

In this paper we restrict to the case of even genus $g=2 k$ and degree $d=k+1$. Then the Brill-Noether number of linear systems of projective dimension $r=1$ and degree $d$ equals $\rho=g-(r+1)(g+r-d)=0$. By the Hurwitz-Zeuthen formula the number of (simple) branch points is $b=6 k$ and the dimension $3 g-3$ of the Hurwitz scheme equals that of $\overline{\mathcal{M}}_{g}$.

There is a natural map $\pi: \bar{H}_{g, d} \rightarrow \overline{\mathcal{M}}_{g}$ with $\overline{\mathcal{M}}_{g}$ the moduli space of stable curves of genus $g$, defined by contracting the unstable rational components of an admissible cover. Moreover, there is also a natural map $q$ of $\bar{H}_{g, d}$ to the moduli space $\overline{\mathcal{M}}_{0, b}$ 
of stable curves of genus 0 with $b$ marked points. The Hurwitz space thus forms a correspondence between $\overline{\mathcal{M}}_{2 k}$ and $\overline{\mathcal{M}}_{0,6 k}$ :

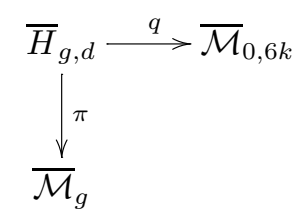

For a general curve $C$ of genus $g=2 k$ the number of $g_{d}^{1}$ 's with $d=k+1$ equals $N=\left(\begin{array}{c}2 k \\ k+1\end{array}\right) / k$, and the natural map $\pi: \bar{H}_{g, d} \rightarrow \overline{\mathcal{M}}_{g}$ is generically finite of degree $(6 k) ! N$.

The boundary $\bar{H}_{g, d}-H_{g, d}$ consists of a finite number of divisors. An irreducible divisor in the boundary of $\bar{H}_{g, d}$ maps under $q$ to an irreducible divisor in the boundary of $\overline{\mathcal{M}}_{0, b}$. The irreducible boundary divisors of $\overline{\mathcal{M}}_{0, b}$ correspond bijectively to the decompositions $\{1, \ldots, b\}=\Lambda \sqcup \Lambda^{c}$ into two disjoint subsets $\Lambda, \Lambda^{c}$, each with at least two elements. We shall write $S^{\Lambda}$ for such a boundary divisor with the rule that $S^{\Lambda}=S^{\Lambda^{c}}$. The generic member of $S^{\Lambda}$ is a stable rational curve with two irreducible components, $\mathbb{P}_{1}$ and $\mathbb{P}_{2}$, meeting in a point $s$ such that the marked points corresponding to $\Lambda$ all lie on one of $\mathbb{P}_{1}$ and $\mathbb{P}_{2}$.

Under the map $\pi: \bar{H}_{g, d} \rightarrow \overline{\mathcal{M}}_{g}$ an irreducible boundary divisor of $\bar{H}_{g, d}$ either maps to the boundary of $\overline{\mathcal{M}}_{g}$ or has a non-empty intersection with $\mathcal{M}_{g}$. We first determine the boundary divisors that map dominantly to an irreducible divisor in the boundary of $\overline{\mathcal{M}}_{g}$; in a later section we determine the irreducible components of these divisors. Recall that the boundary $\overline{\mathcal{M}}_{g}-\mathcal{M}_{g}$ of $\overline{\mathcal{M}}_{g}$ consists of the irreducible divisors $\Delta_{j}$ with $0 \leq j \leq[g / 2]$, where the generic element of $\Delta_{0}$ is an irreducible one-nodal curve and the generic element of $\Delta_{j}$ is a curve with two irreducible components of genus $j$ and $g-j$ meeting in one point.

3. Boundary divisors mapping to the boundary of $\mathcal{M}_{g}$. We determine which divisors in the boundary of $\bar{H}_{g, d}$ with $g=2 k$ and $d=k+1$ map dominantly to an irreducible boundary divisor of $\overline{\mathcal{M}}_{g}$. Please note that by ramification degree at a point we do not mean the ramification index, but the degree of the local covering map at this point.

Proposition 3.1. Let $0 \leq j \leq k$. There are $[j / 2]+1$ boundary divisors $E_{j, c}$ with $c=0, \ldots,[j / 2]$ mapping dominantly to $\Delta_{j}$ under $\pi: \bar{H}_{g, d} \rightarrow \overline{\mathcal{M}}_{g}$.

i) For $j \geq 1$ the divisor $E_{j, c}$ decomposes as $\sum_{\Lambda} E_{j, c}^{\Lambda}$ with $\Lambda$ running over the subsets of $\{1, \ldots, 6 k\}$ of cardinality $3 j$, where we identify $\Lambda$ with $\Lambda^{c}$ if $j=k$. The general element $\varphi: X \rightarrow P$ of $E_{j, c}^{\Lambda}$ maps to a curve $P=\mathbb{P}_{1} \cup \mathbb{P}_{2}$ with $\mathbb{P}_{1}$ (resp. $\mathbb{P}_{2}$ ) carrying the $6 k-3 j$ (resp. $3 j$ ) marked points of $\Lambda^{c}$ (resp. $\Lambda$ ). The inverse image of $\mathbb{P}_{1}$ consists of a smooth curve $C_{1}$ of genus $2 k-j$ and $c$ smooth rational curves $R_{1}, \ldots, R_{c}$, while the inverse image of $\mathbb{P}_{2}$ consists of a smooth curve $C_{2}$ of genus $j$ and $k-j+c$ smooth rational curves $S_{1}, \ldots, S_{k-j+c}$. Each $R_{\mu}$ meets $C_{2}$ in a single point $q_{\mu}$ and each $S_{\nu}$ meets $C_{1}$ in a single point $p_{\nu}$. The curves $C_{1}$ and $C_{2}$ meet in a single point $p$.

The map $\varphi$ restricted to $C_{1}$ (resp. $\left.C_{2}\right)$ has degree $k+1-c($ resp. $j+1-c)$ and has degree 1 on the $R_{\mu}$ and $S_{\nu}$. The $p_{\nu}$ and $q_{\mu}$ are not ramification points, but $p$ has ramification degree $j+1-2 c$ and the points $q_{\mu}, p_{\nu}, p$ all map to $s$. 


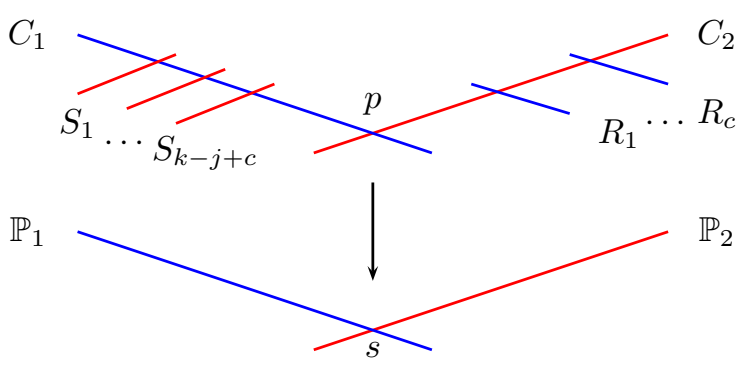

ii) For $j=0$ the divisor $E_{0}=E_{0,0}$ decomposes as $\sum_{\Lambda} E_{0}^{\Lambda}$ with $\Lambda$ running through the 2-element subsets of $\{1, \ldots, 6 k\}$. The general element $\varphi: X \rightarrow P$ of $E_{0}^{\Lambda}$ has $P=\mathbb{P}_{1} \cup \mathbb{P}_{2}$ with $\mathbb{P}_{1}\left(\right.$ resp. $\left.\mathbb{P}_{2}\right)$ carrying the 2 (resp. $\left.6 k-2\right)$ marked points of $\Lambda$ (resp. $\left.\Lambda^{c}\right)$. The inverse image of $\mathbb{P}_{1}$ consists of a smooth rational curve $C_{1}$ and $k-1$ smooth rational curves $R_{1}, \ldots, R_{k-1}$ while the inverse image of $\mathbb{P}_{2}$ is a smooth curve $C_{2}$ of genus $2 k-1$. The curves $C_{1}$ and $C_{2}$ meet in two points $p$ and $q$ and each $R_{\mu}$ meets $C_{2}$ in a single point $p_{\mu}$ that is not a ramification point on $C_{2}$.

The map $\varphi$ restricted to $C_{2}$ has degree $k+1$, while restricted to $C_{1}$ it has degree 2 and degree 1 on the $R_{\mu}$.

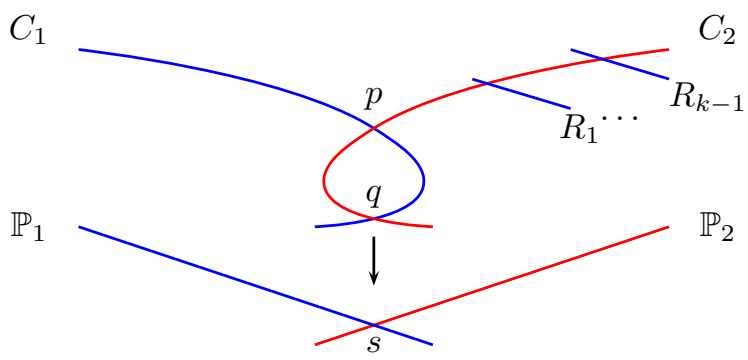

REMARK 3.2. Later we shall prove that the $E_{j, c}^{\Lambda}$ and $E_{0}^{\Lambda}$ are irreducible.

We also need to determine the degree of the restriction of $\pi$ to the divisor $E_{j, c}$.

Proposition 3.3. i) For $j>0$ the degree of the restriction $\pi_{j, c}: E_{j, c} \rightarrow \Delta_{j}$ of $\pi$ to $E_{j, c}$ is

$$
\frac{(6 k) !(j+1-2 c)^{2}}{(j+1)(2 k-j+1)}\left(\begin{array}{c}
j+1 \\
c
\end{array}\right)\left(\begin{array}{c}
2 k-j+1 \\
k+1-c
\end{array}\right) .
$$

ii) For $j=0$ the degree of the restriction $\pi_{0}: E_{0} \rightarrow \Delta_{0}$ of $\pi$ to $E_{0}$ is

$$
\frac{(6 k) !}{2 k}\left(\begin{array}{c}
2 k \\
k+1
\end{array}\right)=\frac{(6 k) !}{2} N
$$

Proof. We shall prove the two propositions 3.1 and 3.3 at the same time. 
i) Suppose $j \geq 1$. We first show that the above loci $E_{j, c}$ in $\bar{H}_{g, d}$ defined by describing their general element are divisors in the boundary of $\bar{H}_{g, d}$. We apply [16], Theorem A, p. 71 [note that there is a misprint in the formulas $\left(^{*}\right)$ there: instead of $h^{0}(L(-2 d-g-1) p) \geq 1$ one should read: $\left.h^{0}(L[-(2 d-g-1)] p) \geq 1\right]$ with $g=j$ (resp. $g=2 k-j)$ and $d=d_{2}=j+1-c$ (resp. $\left.d=d_{1}=k+1-c\right)$. For $g=j$ we have in the notation of loc. cit. $\min d=j / 2+1$ and $\max d=j+1$. Similarly for $g=2 k-j$ we have $\min d=(2 k-j) / 2+1$ and $\max d=k+1 \leq 2 k-j+1$. Hence the range of $d$ satisfies the requirements of the theorem. Observe also that $2 d-g-1=j+1-2 c$. The theorem then implies that the generic pair $\left(C_{2}, p\right)$ with $C_{2}$ of genus $j$ and $p$ a point of $C_{2}$ can be expressed in

$$
a\left(j, d_{2}\right)=\frac{j+1-2 c}{j+1}\left(\begin{array}{c}
j+1 \\
c
\end{array}\right)
$$

ways as a ramified cover of $\mathbb{P}^{1}$ of degree $d_{2}$ with all branch points simple except the image of $p$ over which $p$ is the only ramification point with degree $j+1-2 c$. Similarly, the generic pair $\left(C_{1}, p\right)$ with $C_{1}$ of genus $2 k-j$ and $p \in C_{1}$ can be expressed in

$$
a\left(2 k-j, d_{1}\right)=\frac{j+1-2 c}{2 k-j+1}\left(\begin{array}{c}
2 k-j+1 \\
k+1-c
\end{array}\right)
$$

ways as a ramified cover of $\mathbb{P}^{1}$ of degree $d_{1}$ with all branch points simple except the image of $p$ over which $p$ is the only ramification point with degree $j+1-2 c$. By a dimension count we have now that the locus $E_{j, c}$ is pure of codimension 1 in $\bar{H}_{g, d}$ and hence $E_{j, c}$ defines a divisor.

The degree of the restricted map $\pi_{j, c}: E_{j, c} \rightarrow \Delta_{j}$ is given by

$$
(6 k) ! a\left(j, d_{2}\right) a\left(2 k-j, d_{1}\right)
$$

and this equals

$$
(6 k) ! \frac{j+1-2 c}{j+1}\left(\begin{array}{c}
j+1 \\
c
\end{array}\right) \frac{j+1-2 c}{2 k-j+1}\left(\begin{array}{c}
2 k-j+1 \\
k+1-c
\end{array}\right) .
$$

But by the identity

$$
\sum_{c=0}^{[j / 2]} \frac{(j+1-2 c)^{2}}{(j+1)(2 k-j+1)}\left(\begin{array}{c}
j+1 \\
c
\end{array}\right)\left(\begin{array}{c}
2 k-j+1 \\
k+1-c
\end{array}\right)=\frac{1}{k}\left(\begin{array}{c}
2 k \\
k+1
\end{array}\right)
$$

we have $\sum_{c=0}^{[j / 2]} \operatorname{deg} \pi_{j, c}=\operatorname{deg} \pi=N$ and since $\bar{H}_{g, d}$ is projective and irreducible and $\overline{\mathcal{M}}_{g}$ is projective, irreducible and smooth in codimension 2 , there is no room for other divisors in the boundary mapping dominantly to $\Delta_{j}$.

ii) For $j=0$ the analysis gives that the curve described in the Proposition 3.1 ii) is a general member of a divisor which maps to $\Delta_{0}$. Indeed, in this situation $\rho=1$ and hence the curve $C_{2}$ possesses a $g_{k+1}^{1}$ passing through two generic points: the pre-image of the space $W_{k+1}^{1}$ in $\mathrm{Sym}^{k+1} C_{2}$ is 2-dimensional and hence intersects $p+q+\operatorname{Sym}^{k-1} C_{2}$ (which of class $x^{2}$, where $x$ is the ample class representing the divisor $p+\operatorname{Sym}^{k} C_{2}$, see [1] Ch. VII, Prop. 2.2) for every choice of $p, q$. The maps $C_{i} \rightarrow \mathbb{P}_{i}$ are not ramified at the points $p, q$. Indeed, by the above mentioned Theorem $\mathrm{A}$ in [16], a generic couple $\left(C_{2}, p\right)$, with $g\left(C_{2}\right)=2 k-1$, possesses a finite number of pencils $\gamma$ of degree $k+1$ with $\gamma \geq 2 p$. Therefore, for a generic $q$ there is no such 
pencil with $\gamma \geq 2 p+q$. By [13], Main Theorem 2c, p. 235, there are $\frac{1}{k}\left(\begin{array}{c}2 k \\ k+1\end{array}\right)=N$ distinct such linear systems.

Note that the symmetric group $\mathbb{S}_{6 k}$ does not act freely on $E_{0}$, since we can compose $\pi$ with the automorphism of $P=\mathbb{P}_{1} \cup \mathbb{P}_{2}$ that is the identity on $\mathbb{P}_{2}$, fixes the intersection point $s$ and exchanges the two branch points on $\mathbb{P}_{1}$. This lifts to an automorphism of $C_{1}$ fixing $p$ and $q$ and interchanging the ramification points. Therefore the degree of the restricted map $\pi_{0}: E_{0} \rightarrow \Delta_{0}$ is $\frac{(6 k) !}{2} N$, which is half of the generic degree of the map $\pi: \bar{H}_{d, g} \rightarrow \overline{\mathcal{M}}_{g}$. On the other hand, a local analysis shows, see [16], bottom of p. 76, that the map $\pi$ is simply ramified along the divisor $E_{0}$. This shows that $E_{0}$ is a divisor in the boundary which maps dominantly to $\Delta_{0}$ and there is no room for other divisors.

4. Boundary divisors not mapping to the boundary of $\mathcal{M}_{g}$. We now determine the divisors in the boundary $\bar{H}_{g, d}$ that map dominantly to a divisor in $\overline{\mathcal{M}}_{g}$ that hits $\mathcal{M}_{g}$.

Proposition 4.1. There are two divisors $E_{2}$ and $E_{3}$ in the boundary of $\bar{H}_{g, d}$ that under $\pi$ map dominantly to a divisor in $\overline{\mathcal{M}}_{g}$ that has non-zero intersection with $\mathcal{M}_{g}$. We have a decomposition $E_{2}=\sum_{\Lambda} E_{2}^{\Lambda}$ into $\left(\begin{array}{c}6 k \\ 2\end{array}\right)$ divisors and $\Lambda \subset\{1, \ldots, 6 k\}$ and $\# \Lambda=2$. Similarly, we have a decomposition $E_{3}=\sum_{\Lambda} E_{3}^{\Lambda}$ in $\left(\begin{array}{c}6 k \\ 2\end{array}\right)$ divisors. Their description is as follows.

i) Each general member $\varphi: X \rightarrow P$ of $E_{2}^{\Lambda}$ maps to a curve $P=\mathbb{P}_{1} \cup \mathbb{P}_{2}$ with $\mathbb{P}_{1}$ (resp. $\mathbb{P}_{2}$ ) carrying the 2 (resp. $6 k-2$ ) marked points of $\Lambda$ (resp. $\Lambda^{c}$ ). The inverse image of $\mathbb{P}_{2}$ is a smooth curve $C$ of genus $g$ mapping with degree $k+1$ to $\mathbb{P}_{2}$, while the inverse image of $\mathbb{P}_{1}$ consists of $k-3$ smooth rational curves $R_{1}, \ldots, R_{k-3}$ mapping with degree 1 to $\mathbb{P}_{1}$ and two smooth rational curves $S_{1}, S_{2}$ mapping with degree 2 to $\mathbb{P}_{1}$. The intersection points $q_{i}$ of $S_{i}$ with $C$ are ramification points of degree 2.

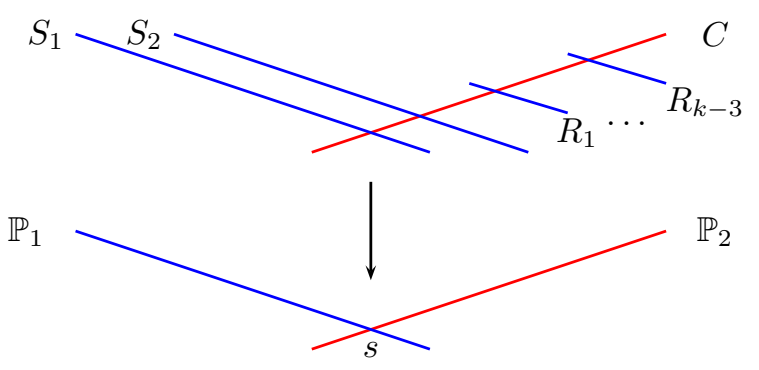

ii) Each general member $\varphi: X \rightarrow P$ of $E_{3}^{\Lambda}$ maps to a curve $P=\mathbb{P}_{1} \cup \mathbb{P}_{2}$ with $\mathbb{P}_{1}$ (resp. $\mathbb{P}^{2}$ ) carrying the 2 (resp. $6 k-2$ ) marked points of $\Lambda$ (resp. $\Lambda^{c}$ ). The inverse image of $\mathbb{P}_{2}$ is a smooth curve $C$ of genus $g$ mapping with degree $k+1$ to $\mathbb{P}_{2}$, while the inverse image of $\mathbb{P}_{1}$ consists of $k-2$ smooth rational curves $R_{1}, \ldots, R_{k-2}$ mapping with degree 1 to $\mathbb{P}_{1}$ and one smooth rational curve $S$ mapping with degree 3 to $\mathbb{P}_{1}$. The intersection point $q$ of $S$ with $C$ is a ramification point of degree 3 . 

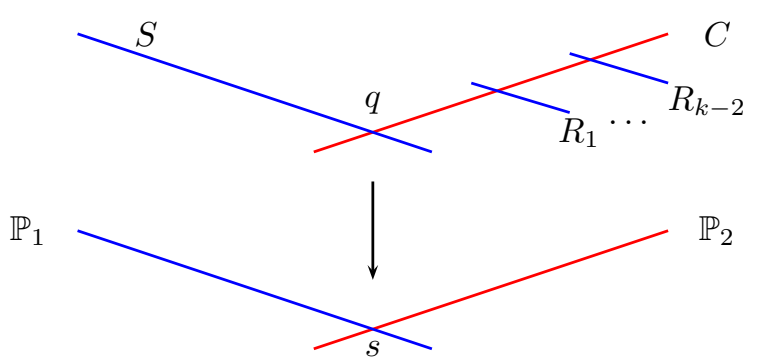

Proof. If we want the image of an admissible cover to be a smooth curve of genus $g$ we must have over (say) $\mathbb{P}_{2}$ a smooth curve of genus $g=2 k$ and no rational components. Indeed, otherwise the restriction of the covering map on $C$ has degree $\leq k=d-1$. But then $\rho \leq-2$ and hence the image cannot be a divisor, see [6], Thm. 1.1. Over $\mathbb{P}_{1}$ we then have only rational curves. A naive dimension count shows that the number of branch points on $\mathbb{P}_{2}$ outside the singular point should be $b-2$ and hence on $\mathbb{P}_{1}$ there should be 2 . In fact, in this case the total number of branch points on $\mathbb{P}_{2}$ is $b-1$ and hence the number of parameters for the curve $C$ is $b-1-3=6 k-4=3 g-4$ as required (and this is the only case where this happens). Then, over $\mathbb{P}_{1}$ only two cases are possible, namely the ones described in the statement of the proposition, see also [15], p. 181-83 and Figures 3.146 on p. 177, and 3.154 on p. 183 (the first case corresponds to the situation where two branch points come together but the two ramification points remain distinct points on the same fiber and the second to the case where the two ramification points come together too). Note that in the first case each of the $S_{i}$ contain one marked point not mapping to $s$.

REMARK 4.2. For later use we notice that the Hurwitz number of degree 3 covers of $\mathbb{P}^{1}$ of genus 0 with one triple ramification point and two simple branch points is 1 . The involution on $\mathbb{P}^{1}$ fixing the triple branch point and interchanging the other two branch points lifts to the cover. Similarly, the Hurwitz number of degree 2 covers of genus 0 with two branch points is 1 and the involution interchanging the two branch points and fixing a third point lifts to the cover.

LEMMA 4.3. The formal local ring that pro-represents the infinitesimal deformations of a general point of $E_{3}^{\Lambda}$ is smooth, but for a general point of $E_{2}^{\Lambda}$ it equals

$$
\mathbb{C}\left[\left[t_{11}, t_{12}, t_{2}, \ldots, t_{b-3}\right]\right] /\left\langle t_{11}^{2}-t_{12}^{2}\right\rangle \text {. }
$$

Proof. The result for $E_{3}^{\Lambda}$ follows from [16], p. 62. For each general point of $E_{2}^{\Lambda}$ the complete local ring pro-representing the infinitesimal deformations equals

$$
\mathbb{C}\left[\left[t_{1}, \ldots, t_{b-3}, t_{11}, t_{12}\right]\right] /\left\langle t_{11}^{2}-t_{1}, t_{12}^{2}-t_{1}\right\rangle \cong \mathbb{C}\left[\left[t_{11}, t_{12}, t_{2}, \ldots, t_{b-3}\right]\right] /\left\langle t_{11}^{2}-t_{12}^{2}\right\rangle .
$$

5. The trace curve of a pencil. In this section we collect a few results about trace curves that we need in the sequel. Let $C$ be smooth curve of genus $g$ together 
with a pencil (a linear system of projective dimension 1 ) of degree $d$, say $\gamma$. We define the trace curve of $\gamma$ by

$$
T_{\gamma}=\{(p, q) \in C \times C: \gamma \geq p+q\} .
$$

Here by $\gamma \geq p+q$ we mean that there is an effective divisor in $\gamma$ containing $p$ and $q$. The following lemma gives information on the singularities that $T_{\gamma}$ might have. In the following we shall assume that our pencils are base point free.

LEMMA 5.1. If $\gamma$ is base-point free then $T_{\gamma}$ is smooth except for possible singularities at points $(p, q)$ with both $p$ and $q$ ramification points of $\gamma$. A point $(p, p)$ with $p$ a ramification point of order $m$ (of the map to $\mathbb{P}^{1}$ ) gives an ordinary singularity of order $m-1$ on $T_{\gamma}$. Morover, if $(p, q) \in T_{\gamma}$ with $p \neq q$ and $p$ and $q$ simple ramification points then the singularity of $T_{\gamma}$ at $(p, q)$ is a simple node.

Proof. Let $\{f, g\}$ be a basis of the pencil $\gamma$ and let $(p, p)$ be a point of $T_{\gamma}$ and let $z$ be a local coordinate at $p$. Then $T_{\gamma}$ is locally at $(p, p)$ given by $h=0$ with

$$
h\left(z_{1}, z_{2}\right)=\frac{f\left(z_{1}\right) g\left(z_{2}\right)-f\left(z_{2}\right) g\left(z_{1}\right)}{z_{1}-z_{2}} .
$$

We may assume that $\operatorname{ord}_{p}(f)=m>0$ and $\operatorname{ord}_{p}(g)=0$. Write $f=z^{m} f_{1}$ and find in the local ring

$$
h\left(z_{1}, z_{2}\right)=\frac{z_{1}^{m}-z_{2}^{m}}{z_{1}-z_{2}} f_{1}(0) g(0),
$$

so locally at $(p, p)$ the curve $T_{\gamma}$ consists of $m-1$ branches passing transversally through $(p, p)$.

If $(p, q) \in T_{\gamma}$ with $p \neq q$ and $z$ (resp. $w$ ) a local coordinate at $p$ (resp. $q$ ) we write $f=f_{1}(z)$ and $f=f_{2}(w)$ and similarly $g=g_{1}(z)$ and $g=g_{2}(w)$ in the local rings of $p$ and $q$. The equation of $T_{\gamma}$ is then $h(z, w)=f_{1}(z) g_{2}(w)-f_{2}(w) g_{1}(z)=0$. Write $f_{1}=a_{0}+a_{1} z+\ldots$ and $g_{1}=b_{0}+b_{1} z+\ldots$; furthermore $f_{2}=c_{0}+c_{1} w+\ldots$ and $g_{2}=d_{0}+d_{1} w+\ldots$ and find that a singularity at $(p, q)$ means that besides $a_{0} d_{0}-b_{0} c_{0}=0$ we have $a_{1} d_{0}-b_{1} c_{0}=0$ and $a_{0} d_{1}-b_{0} c_{1}=0$. We may assume that $a_{0}=0$ and $b_{0} \neq 0$, hence $c_{0}=0$ and $d_{0} \neq 0$, so that a singularity means $a_{1}=c_{1}=0$, i.e. both $p$ and $q$ are ramification points. Then the next term in $h$ is $a_{2} d_{0} z^{2}-c_{2} b_{0} w^{2}$ and this shows that if $a_{2}$ and $c_{2}$ do not vanish we have a simple node.

Lemma 5.2. Let $\gamma$ be a base point free $g_{d}^{1}$ with all branch points simple except one with arbitrary ramification. Then $T_{\gamma}$ is irreducible.

Proof. Consider the map $T_{\gamma} \rightarrow \mathbb{P}^{1}$ defined as the composition of the first projection $T_{\gamma} \rightarrow C$ composed with the map $C \rightarrow \mathbb{P}^{1}$ defined by $\gamma$. All singular points and all the ramification points of $T_{\gamma} \rightarrow \mathbb{P}^{1}$ lie over the branch points of $C \rightarrow \mathbb{P}^{1}$. So for both coverings we consider the same monodromy group ( $\pi_{1}$ of the punctured line). Since $T_{\gamma} \subset C \times C$ the monodromy action for $T_{\gamma}$ is induced by the monodromy action for $C$. By showing that the latter is doubly transitive the result will follow. Since $C \rightarrow \mathbb{P}^{1}$ is simply branched except possibly at one point the monodromy is generated by the transpositions at the simple branch points (since the product of the permutations of all branch points is 1 ). We thus see that this generates a transitive subgroup of $\mathbb{S}_{d}$, hence it is the whole symmetric group and therefore doubly transitive.

Corollary 5.3. The trace curves induced by the pencils on $C_{1}, C_{2}$ as in i) of Proposition 3.1 and on $C$ as in ii) of Proposition 4.1 have one singular point which 
is an ordinary singularity and lies on the diagonal. The trace curve induced by the pencil on $\mathrm{C}_{2}$ as in ii) of Proposition 3.1 is smooth. The trace curve induced by the pencil on $C$ as in i) of Proposition 4.1 has two nodal singularities at two symmetric points. Moreover all the above trace curves are irreducible.

6. An irreducibility result. In this section we shall prove that the boundary divisors $E_{j, c}^{\Lambda}$ defined in Proposition 3.1 and the boundary divisors $E_{3}^{\Lambda}$ defined in Proposition 4.1 are irreducible and that $E_{2}^{\Lambda}$ maps $2: 1$ to an irreducible space. We start with the result for $E_{2}$ and $E_{3}$.

Let $\underline{c}$ be a conjugacy class of the symmetric group $\mathbb{S}_{d}$ on $d$ objects. It is given by a partition of $d$. We consider the Hurwitz space $\mathcal{H}_{d, b, \underline{c}}$ parametrizing isomorphism classes of (connected) Riemann surfaces that are degree $d$ covers of $\mathbb{P}^{1}$ that are simply branched at $b$ (unordered) points of the projective line different from infinity and have ramification type $\underline{c}$ over infinity. This has the structure of a smooth analytic space; this may be proved as in [10].

We define $\Pi_{b}:=\left(\mathbb{C}^{1}\right)^{\times b}-\Delta$ with $\Delta$ the big diagonal and $\Sigma_{b}:=\operatorname{Sym}^{b} \mathbb{C}-D$, with $D$ the discriminant locus and then have a natural map $p: \Pi_{b} \rightarrow \Sigma_{b}$.

There is a natural covering map $\mu: \mathcal{H}_{d, b, \underline{c}} \rightarrow \Sigma_{b}$ by assigning to each point of $\mathcal{H}_{d, b, \underline{c}}$ the set of $b$ points with simple branching. We get a projection map

$$
\operatorname{pr}_{2}: \mathcal{H}_{d, b, \underline{c}} \times_{\Sigma_{b}} \Pi_{b} \rightarrow \Pi_{b} .
$$

Let now $H_{d, b, c}$ be the Hurwitz space parametrizing isomorphism classes of (connected) Riemann surfaces that are degree $d$ covers of $\mathbb{P}^{1}$ simply branched at $b$ ordered points of the projective line and have an extra point with ramification of type $\underline{c}$, modulo the equivalence relation that two such covers $f_{i}: C_{i} \rightarrow \mathbb{P}^{1}$ are equivalent if there exist isomorphisms $h: C_{1} \rightarrow C_{2}$ and $\gamma: \mathbb{P}^{1} \rightarrow \mathbb{P}^{1}$ with $f_{2} \circ h=\gamma \circ f_{1}$.

There is a natural surjective map

$$
m: \mathcal{H}_{d, b, \underline{c}} \times_{\Sigma_{b}} \Pi_{b} \rightarrow H_{d, b, \underline{c}},
$$

given by associating to the cover $f: C \rightarrow \mathbb{P}^{1}$ and a set of ordered branch points $\left\{a_{1}, \ldots, a_{b}\right\}$ the cover with its ordered branch points.

THEOREM 6.1. With the notations as before and $\underline{c}$ the conjugacy class of $\varphi=$ (12)(34) or of $\varphi=(123)$ the Hurwitz space $H_{d, b, \underline{c}}$ is irreducible.

Corollary 6.2. The divisor $E_{3}^{\Lambda}$ is irreducible. The divisor $E_{2}^{\Lambda}$ is a twofold cover of an irreducible Hurwitz space.

We first deduce the corollary from the theorem. With $\underline{c}$ the type of a 3-cycle, say (123), and with $b=6 k-2$ we have a natural inclusion $\nu: H_{d, b, \underline{c}} \rightarrow E_{3}^{\Lambda}$ given as follows. A point of $H_{d, b, \underline{c}}$ corresponds to a cover $C_{2} \rightarrow \mathbb{P}_{2}$ of the projective line with an ordering of the $6 k-2$ branch points (which we assume to be indexed by the set $\Lambda^{c}$ ). Then $\nu$ sends this point to the point of $E_{3}^{\Lambda}$ corresponding to the admissible cover $X \rightarrow P=\mathbb{P}_{1} \cup \mathbb{P}_{2}$, with $\mathbb{P}_{1}$ containing the marked points $p_{i}$ with $i \in \Lambda$ and $X$ the curve with $C_{2}$ over $\mathbb{P}_{2}$, while over $\mathbb{P}_{1}$ we have a union of rational curves attached at the ramification points of $C_{2}$ over infinity with the appropriate ramification conditions. Note that the positions of the two points $p_{i}, i \in \Lambda$, on $\mathbb{P}_{1}$ do not matter because of the automorphism group of $\mathbb{P}^{1}$, cf. also Remark 4.2. This is a dominant map since its image contains the general member of $E_{3}^{\Lambda}$. Since $H_{d, b, \underline{c}}$ is irreducible we conclude that $E_{3}^{\Lambda}$ is irreducible. Similarly for $E_{2}^{\Lambda}$, but here we use a map to the Hurwitz space, 
by mapping a generic point of $E_{2}^{\Lambda}$ to the corresponding point of $H_{d, b, \underline{c}}$; but here we have to take into account the marking of the two ramification points of degree 2 on $C_{2}$ lying over the same point. So the map is of degree 2 .

Remark 6.3. The degree 2 rational map $E_{2}^{\Lambda} \rightarrow \mathcal{M}_{g}$ factors through $H_{d, b, \underline{c}}$. We do not know whether the map $E_{2}^{\Lambda} \rightarrow H_{d, b, \underline{c}}$ is split or not.

Proof. We prove the theorem by showing that the monodromy of $\mathrm{pr}_{2}$ acts transitively on the fibres and this implies that the fibre product $\mathcal{H}_{d, b, c} \times{ }_{\Sigma_{b}} \Pi_{b}$ is connected and by the smoothness it is then irreducible and therefore its image $H_{d, b, \underline{c}}$ is irreducible too.

We choose a point $A \in \Sigma_{b}$ and a point of $\Pi_{b}$ mapping to $A$ under $p$. That is, we order the points of $A$, say $A=\left\{a_{1}, \ldots, a_{b}\right\}$. The points of the fiber $\mu^{-1}(A)$ correspond to the $\mathbb{S}_{d}$-conjugacy classes of $b$-tuples $\left[t_{1}, \ldots, t_{b}\right]$ with $t_{i}$ a transposition in the symmetric group $\mathbb{S}_{d}$ such that these generate $\mathbb{S}_{d}$ and such that the product $t_{1} \cdots t_{b}$ has type $\underline{c}$.

By fixing a permutation $\varphi$ from the conjugacy class $\underline{c}$ we can then describe the fibre $\mu^{-1}(A)$ as the quotient

$$
\Xi_{\varphi}^{d, b} / G_{\varphi}
$$

where $G_{\varphi} \subset \mathbb{S}_{d}$ is the stabilizer of $\varphi$ under conjugation and $\Xi_{\varphi}^{d, b}$ is the set

$$
\Xi_{\varphi}^{d, b}=\left\{\left[t_{1}, \ldots, t_{b}\right], t_{i} \text { are transpositions generating } \mathbb{S}_{d}, t_{1} \cdots t_{b}=\varphi\right\}
$$

on which $G_{\varphi}$ acts by conjugation.

According to [17], Theorem 1, the braid group $B_{b}=\pi_{1}\left(\Sigma_{b}, A\right)$ acts transitively on $\Xi_{\varphi}^{d, b}$. We consider now the two cases, $\varphi=(123)$ and $\varphi=(12)(34)$ and we prove that in both cases the pure braid group $P_{b}=\pi_{1}\left(\Pi_{b},\left\{a_{1}, \ldots, a_{b}\right\}\right)$ acts transitively on $\Xi_{\varphi}^{d, b}$. Note that $B_{b} / P_{b} \cong \mathbb{S}_{b}$.

We work as in [4], proof of Lemma 3.2. We denote by $\Gamma_{i}, i=1, \ldots, b-1$, the standard generators of the braid group $B_{d}$. The action of $\Gamma_{i}$ on $\Xi_{\varphi}^{d, b}$ sends $\left[t_{1}, \ldots, t_{i}, t_{i+1}, \ldots, t_{b}\right]$ to $\left[t_{1}, \ldots, t_{i+1}, t_{i+1} t_{i} t_{i+1}, \ldots, t_{b}\right]$. Moreover, $\Gamma_{i}$ interchanges the points $a_{i}$ and $a_{i+1}$. We examine now separately the two cases:

Case i: $\varphi=(123)$. We start with the element $\left[t_{1}, \ldots, t_{b}\right] \in \Xi_{\varphi}^{d, b}$. By the above transitivity result we can find an element $\Gamma$ of $B_{b}$ which sends $\left[t_{1}, \ldots, t_{b}\right]$ to the following element of $\Xi_{\varphi}^{d, b}$ :

$$
\sigma_{0}=[(13),(12),(14),(14), \ldots,(1 d-1),(1 d-1),(1 d), \ldots,(1 d)],
$$

where the transpositions $(14), \ldots,(1 d-1)$ occur in pairs and the last transposition (1d) appears $b-2(d-3)$ times (which by the Hurwitz-Zeuthen formula is an even number).

We now consider the elements $\Gamma_{i}^{3}, i=1, \ldots, b-1$. The action by such an element interchanges $a_{i}$ and $a_{i+1}$ and so the above set of elements acts transitively on the permutation group $\mathbb{S}_{b}$ of the indices. On the other hand we observe that it acts trivially on $\sigma_{0}$, because the supports of two consecutive transpositions in $\sigma_{0}$ have a common part: if $[(m n),(k l)]$ denote the $i$ th and $(i+1)$ th element in $\sigma_{0}$, then if $(m n)=(k l)$ the action of $\Gamma_{i}$ is trivial, and if $n=k$ but $n \neq m \neq l$ then the action of $\Gamma_{i}^{3}$ is given by

$$
[(m n),(n l)] \rightarrow[(n l),(m l)] \rightarrow[(m l),(m n)] \rightarrow[(m n),(n l)] .
$$


Because of the transitivity of the action of the set $\Gamma_{i}^{3}, i=1, \ldots, b-1$, on $\mathbb{S}_{b}$ we may compose $\Gamma$ with an appropriate sequence of the elements $\Gamma_{i}^{3}$ so that the composition belongs to the pure braid group $P_{b}$ and the action still sends our $b$-tuple $\left[t_{1}, \ldots, t_{b}\right]$ to the fixed element $\sigma_{0}$. This proves that $P_{b}$ acts transitively on $\Xi_{\varphi}^{d, b}$, with $\varphi=(123)$.

Case ii): $\varphi=(12)(34)$. We work as before with

$$
\sigma_{0}=[(12),(13),(13),(34),(14),(14), \ldots,(1 d-1),(1 d-1),(1 d), \ldots,(1 d)]
$$

where the last transposition $(1 d)$ appears $b-2(d-2)$ times (which is an even number).

This proves that in the two cases the product $\mathcal{H}_{d, b, \underline{c}} \times{ }_{\Sigma_{b}} \Pi_{b}$ is connected.

Proposition 6.4. Each divisor $E_{j, c}^{\Lambda}$ as in Proposition 3.1 is irreducible.

Proof. The irreducibility of the divisors $E_{j, c}^{\Lambda}$ is proved in a way similar to the case $E_{3}$. With $d_{1}=k+1-c, b_{1}=6 k-3 j$ and $\varphi=(12 \ldots j+1-2 c)$ we define $\sigma_{0}=\left[t_{1}, \ldots, t_{6 k-3 j}\right]$ by taking $t_{\nu}=(1, j+2-2 c-\nu)$ for $\nu=1, \ldots, j-2 c$, and $t_{j-2 c+2 \mu+1}=t_{j-2 c+2 \mu+2}=(1, j+2-2 c+\mu)$ for $\mu=0, \ldots, k-j+c-1$ and the remaining $t_{\nu}$ are equal to $(12)$, i.e. $\sigma_{0}$ is equal to

$$
\left[(1, j+1-2 c), \ldots,(12),(1, j+2-2 c),(1, j+2-2 c), \ldots,\left(1 d_{1}\right),\left(1 d_{1}\right),(12), \ldots,(12)\right] .
$$

Note that $\sigma_{0}$ contains all the transpositions $(1 k), k=1, \ldots d_{1}=k+1-c$, hence it generates the symmetric group $\mathbb{S}_{d_{1}}$. The last transposition (12) appears $[6 k-3 j]-$ $[(j-2 c)+2(k-j+c)]$ times which is the even number $4 k-2 j$. Hence the product of the transpositions contained in $\sigma_{0}$ is $\varphi$. With $\underline{c}$ the type of $\varphi$, an argument similar to the case $E_{3}$ shows that the corresponding Hurwitz space $H_{d_{1}, b_{1}, \underline{c}}$ with ordered branch points is irreducible.

Similarly we show that the Hurwitz space $H_{d_{2}, b_{2}, c}$ is irreducible with $d_{2}=j+1-c$, $b_{2}=3 j$, and $\underline{c}$ the type of $\varphi=(12 \ldots j+1-2 c)$, by defining $\sigma_{0}=\left[t_{1}, \ldots, t_{3 j}\right]$ with $t_{1}, \ldots, t_{j-2 c}$ as in the preceding paragraph and $t_{j-2 c+2 \mu+1}=t_{j-2 c+2 \mu+2}=$ $(1, j+2-2 c+\mu)$ for $\mu=0, \ldots, c-1$ and by setting $t_{\mu}=(12)$ for the remaining indices. The last transposition (12) appears an even number $3 j-[(j-2 c)+2 c]=2 j$ of times. Therefore the space $H_{d_{1}, b_{1}, \underline{c}} \times H_{d_{2}, b_{2}, \underline{c}}$ is irreducible.

We now define the inclusion $\nu: H_{d_{1}, b_{1}, \underline{c}} \times \bar{H}_{d_{2}, b_{2}, \underline{c}} \rightarrow E_{j, c}^{\Lambda}$ as follows: We assume that the $b_{1}=6 k-3 j$ marked points of $H_{d_{1}, b_{1}, \underline{c}}$ take values in the set $\Lambda^{c}$ and the $b_{2}=3 j$ marked points of $H_{d_{2}, b_{2}, \underline{c}}$ take values in the set $\Lambda$. A point $h_{1} \in H_{d_{1}, b_{1}, \underline{c}}$ (resp. $h_{2} \in H_{d_{2}, b_{2}, c}$ ) corresponds to a curve $C_{1}$ (resp. $C_{2}$ ) of genus $2 k-j$ (resp. $j$ ) with a $g_{d_{1}}^{1}$ (resp. $g_{d_{2}}^{1}$ ) having simple branching except in one fiber which has a point $p_{1}$ (resp. $p_{2}$ ) of ramification degree $j+1-2 c$ and simple ramification everywhere else. We then define $\nu\left(h_{1}, h_{2}\right)$ to be the admissible cover $X$ constructed by the above data as in Proposition $3.1 \mathrm{i}$ ) by joining the curves $C_{1}$ and $C_{2}$ at the points $p_{1}$ and $p_{2}$ respectively and attaching rational tails appropriately. The map $\nu$ is a dominant map since its image contains the general member of $E_{j, c}^{\Lambda}$. Since $H_{d_{1}, b_{1}, \underline{c}} \times H_{d_{2}, b_{2}, \underline{c}}$ is irreducible we conclude that $E_{j, c}^{\Lambda}$ is irreducible.

For the divisor $E_{0}$ we have a decomposition $E_{0}=\sum_{\Lambda} E_{0}^{\Lambda}$ with $\Lambda$ running over the subsets of $\{1, \ldots, 6 k\}$ with 2 elements. We prove that $E_{0}^{\Lambda}$ is irreducible.

Proposition 6.5. The divisor $E_{0}^{\Lambda}$ as in Proposition 3.1 ii) is irreducible.

Proof. Consider the Hurwitz space $H_{k+1,6 k-2}$ parametrizing isomorphism classes of (connected) Riemann surfaces of genus $2 k-1$ that are degree $k+1$ covers of $\mathbb{P}^{1}$ simply branched at $6 k-2$ ordered points of the projective line. Let $\mathcal{C}_{H}$ be the universal 
curve over $H_{k+1,6 k-2}$. On $\mathcal{C}_{H} \times_{H_{k+1,6 k-2}} \mathcal{C}_{H}$ we consider the universal trace curve $\mathcal{T}$; the fiber $\mathcal{T}_{h}$ of $\mathcal{T}$ over a point $h \in H_{k+1,6 k-2}$ is the trace curve $\{(x, y) \in C \times C: x+y \leq$ $\gamma\}$ corresponding to $\gamma$, the $g_{k+1}^{1}$ associated to $h$. The curve $\mathcal{T}_{h}$ is an irreducible curve because the $g_{k+1}^{1}$ has simple branching, see section 5 . Therefore $\mathcal{T}$ is an irreducible space. We now define a natural 2:1 map $\nu: \mathcal{T} \rightarrow E_{0}^{\Lambda}$ as follows. We let the $6 k-2$ branch points of $C$ take values in the set $\Lambda^{c}$. A point $h$ of $\mathcal{T}$ corresponds to a curve $C$ with a $g_{k+1}^{1}$ as above, say $\gamma$, and a couple $(p, q)$ of points of $C$ with $\gamma \geq p+q$. We then define $\nu(h)$ to be the admissible cover as in Proposition 3.1 ii), with $C_{2}=C$ and the points $p, q$ as above. We attach to $C_{2}$ the rational curves $C_{1}$ and $R_{1}, \ldots, R_{k-1}$ as in Proposition 3.1. As in the case of $E_{3}$ the position of the branch points on $\mathbb{P}_{1}$ does not matter. The map $\nu$ is a dominant map since its image contains the general member of $E_{0}^{\Lambda}$. Since $\mathcal{T}$ is irreducible we conclude that $E_{0}^{\Lambda}$ is irreducible.

7. The degree of $\pi$ restricted to $E_{3}$ and $E_{2}$. We shall denote the image of the divisor $E_{3}$ (resp. $E_{2}$ ) under the morphism $\pi: \bar{H}_{2 k, k+1} \rightarrow \overline{\mathcal{M}}_{2 k}$ by $D_{3}$ (resp. $D_{2}$ ). We know that $E_{3}$ decomposes as a union of $\left(\begin{array}{c}6 k \\ 2\end{array}\right)$ irreducible divisors $E_{3}^{\Lambda}$, with $\# \Lambda=2$ and similarly $E_{2}=\sum_{\Lambda} E_{2}^{\Lambda}$ with $\left(\begin{array}{c}6 k \\ 2\end{array}\right)$ components (cf. Remark 6.3). It follows from the results of the preceding section that the degree of $\pi: E_{3}^{\Lambda} \rightarrow D_{3}$ (resp. $\pi: E_{2}^{\Lambda} \rightarrow D_{2}$ ) is the same as (resp. is twice) the degree of a map $H_{k+1,6 k-2, \underline{c}} \rightarrow D_{3}$ with $\underline{c}$ the type of a 3 -cycle (resp. of a cycle of type (12)(34)). In fact, the Hurwitz space $H_{k+1,6 k-2, \underline{c}}$ can be identified with the Hurwitz space $H_{k+1,6 k-2,3}$ (resp. $H_{k+1,6 k-2,2+2}$ ), that parametrizes $k+1$ coverings $C \rightarrow D$ with $D$ a $6 k-1$ pointed curve $\left(D, p_{1}, \ldots, p_{6 k-1}\right)$ of genus 0 and $C$ a connected smooth curve of genus $2 k$ which has over $p_{1}$ one point of triple ramification (resp. two simple ramification points) and is simply branched at the points $p_{2}, \ldots, p_{6 k-1}$ and unramified everywhere else. We know that $H_{k+1,6 k-2,3}$ (resp. $H_{k+1,6 k-2,2+2}$ ) is irreducible and hence its compactification $\bar{H}_{k+1,6 k-2,3}$ (resp. $\bar{H}_{k+1,6 k-2,2+2}$ ) by admissible covers (see [3], Section 5) is irreducible.

TheOREM 7.1. The degree of $\pi$ restricted to $E_{3}$ is $(6 k) ! / 2$. The degree of $\pi$ restricted to $E_{2}$ is $(6 k)$ !.

In view of the discussion above it suffices to prove that the degree of the map $\bar{H}_{k+1,6 k-2,3} \rightarrow D_{3}$ (resp. $\bar{H}_{k+1,6 k-2,2} \rightarrow D_{2}$ ) equals $(6 k-2)$ !, in other words that the degree is 1 modulo the action of $\mathbb{S}_{6 k-2}$. Since $\bar{H}_{k+1,6 k-2,3}$ is irreducible it suffices to find an appropriate smooth point of $D_{3}$ and determine the degree of the fiber over this point.

For this we consider linear systems $g_{k+1}^{1}$ on a generic curve of genus $2 k-1$ with $6 k-4$ simple branch points and one branch point over which there is one triple ramification point (resp. two double ramification points). We call such a pencil of degree $k+1$ of type (3) (resp. of type $(2,2)$ ).

Recall that according to Harris ([14], Thm 2.1) for a general curve $C^{\prime}$ of genus $2 k-1$ the number of pencils of degree $k+1$ and of type (3) is finite and equals

$$
b(k)=12 \frac{k-1}{k}\left(\begin{array}{c}
2 k \\
k+1
\end{array}\right) .
$$

Similarly, by the same result (cf. loc. cit.) for a general $C^{\prime}$ of genus $2 k-1$ and a general point $p$ on $C^{\prime}$ there are finitely many pencils $\gamma$ of degree $k+1$ on $C^{\prime}$ with the property that $\gamma \geq 2 p$. Their number equals

$$
a(k)=\frac{1}{k}\left(\begin{array}{c}
2 k \\
k+1
\end{array}\right) .
$$


Moreover, for a general $C^{\prime}$ of genus $2 k-1$ and a general point $p$ the number of pairs $(\gamma, q)$ with $\gamma$ a pencil of degree $k+1$ and $\gamma \geq p+2 q$ is finite and equals

$$
c(k)=5 \frac{k-1}{k}\left(\begin{array}{c}
2 k \\
k+1
\end{array}\right) .
$$

Lemma 7.2. Let $C^{\prime}$ be a general curve of genus $2 k-1$ and let $p$ be a general point of $C^{\prime}$. Then there exists a point $q$ on $C^{\prime}$ such that

1. there exists a unique pencil $\gamma$ on $C^{\prime}$ of degree $k+1$ and type (3) with $\gamma \geq p+q$;

2. there does not exist a pencil $\gamma^{\prime}$ on $C^{\prime}$ of degree $k+1$ with $\gamma^{\prime} \geq 2 p+q$ or with $\gamma^{\prime} \geq p+2 q$.

Proof. Let $\gamma_{i}$ with $i=1, \ldots, b(k)$ be the type (3) pencils of degree $k+1$ and let $T_{3}=\cup_{i=1}^{b(k)} T_{\gamma_{i}}$ be the union of the trace curves associated to the $\gamma_{i}$. Note that by Lemma 5.2 each $T_{\gamma_{i}}$ is irreducible and contained in $C^{\prime} \times C^{\prime}$ and thus $T_{3}$ has a projection $\tau_{3}: T_{3} \rightarrow C^{\prime}$ to the first factor. We now choose a pair $(p, q)$ in $T_{3}$ which is sufficiently general; this means that $p$ is not contained in the image under $\tau_{3}$ of any multiple point of $T_{3}$ and $p$ is not contained in a fibre of a $\gamma_{i}$ containing a ramification point of $\gamma_{i}$; in other words $\# \tau_{3}^{-1}(p)=k b(k)$.

We set $\Sigma_{p}=\tau_{3}^{-1}(p)$. The above $p$ is a general point on $C^{\prime}$. We consider the pencils $\gamma_{1}^{\prime}, \ldots, \gamma_{a(k)}^{\prime}$ of degree $k+1$ on $C^{\prime}$ with $\gamma_{i}^{\prime} \geq 2 p$. Let

$$
\Sigma_{p}^{\prime}=\left\{r^{\prime} \in C^{\prime}: \gamma_{i}^{\prime} \geq 2 p+r^{\prime} \text { for some } 1 \leq i \leq a(k)\right\} .
$$

Then by the result of Harris ([14], p. 44) we have $\# \Sigma_{p}^{\prime}=(k-1) a(k)$ and moreover, if we define

$$
\Sigma_{p}^{\prime \prime}=\left\{r^{\prime \prime} \in C^{\prime}: \gamma_{i}^{\prime} \geq p+2 r^{\prime \prime} \text { for some } 1 \leq i \leq c(k)\right\}
$$

we have by the shape of $a(k), b(k)$ and $c(k)$ that $\# \Sigma_{p}>\# \Sigma_{p}^{\prime}+\# \Sigma_{p}^{\prime \prime}$. Then we can choose a point $q$ in $\Sigma_{p}-\left(\Sigma_{p}^{\prime} \cup \Sigma_{p}^{\prime \prime}\right)$ and by taking for $\gamma$ the unique $\gamma_{i}$ such that $(p, q) \in T_{\gamma_{i}}$ the pencil $\gamma$ and the points $p$ and $q$ satisfy the conditions of our lemma. $\mathbf{Q}$

Note the similarity of the argument with considerations of Harris in [14], p. 458.

We now work out the case of $E_{3}$. After completing that case we give the modifications in the proof to make it work for $E_{2}$ too.

We now take a generic curve $C^{\prime}$ of genus $2 k-1$ and a pencil of degree $k+1$ of type (3), say $\gamma$, on $C^{\prime}$ and a couple of points $p, q$ as in the lemma. Then the nodal curve $C=C^{\prime} /(p \sim q)$ determines a point $[C]$ of $\overline{\mathcal{M}}_{g}$ with $g=2 k$ and this point lies on the divisor $\Delta_{0}$.

Proposition 7.3. The set-theoretic fibre of the map $\pi^{\prime}: \bar{H}_{k+1,6 k-2,3} / \mathbb{S}_{6 k-2} \rightarrow$ $D_{3}$ over the point $[C]$ consists of one point.

Proof. We first describe the admissible cover that represents the unique point of the fibre. It is the admissible cover $X \rightarrow \mathbb{P}_{1} \cup \mathbb{P}_{2}$ with $\mathbb{P}_{1} \cup \mathbb{P}_{2}$ the rational curve consisting of two copies of $\mathbb{P}^{1}$ intersecting transversally in one point $s$. Over $\mathbb{P}_{2}$ the curve $X$ has a component $C^{\prime}$ with a covering $C^{\prime} \rightarrow \mathbb{P}_{2}$ determined by $\gamma$ and the fibre over $s$ contains $p$ and $q$. Over $\mathbb{P}_{1}$ the curve $X$ is the union of a rational curve $R$ which is a double cover of $\mathbb{P}_{1}$ intersecting $C^{\prime}$ at the points $p$ and $q$ with no ramification at these points and having two simple marked branch points and $k-1$ rational curves $R_{i}$ mapping isomorphically to $\mathbb{P}_{1}$ and intersecting $C^{\prime}$ at the remaining $k-1$ points of the fibre over $s$ different from $p$ and $q$. 
We now analyze the uniqueness. The locus of $[C]$ as constructed above has dimension $6 k-5$ : the curve $C^{\prime}$ is generic of genus $2 k-1$ so it contributes $6 k-6$ to the dimension and the pair $(p, q)$ is a generic point of the trace curve $T_{\gamma}$ in $C^{\prime} \times C^{\prime}$ so it contributes 1 . (Note that $p$ was chosen general on $C$ and that results in finitely many choices for $q$.) Since the locus of the admissible covers in $\bar{H}_{k+1,6 k-2,3}$ mapping to a rational curve with more than two components has dimension $\leq 6 k-6$ we conclude that an admissible cover in $\bar{H}_{k+1,6 k-2,3}$ mapping to $[C]$ will correspond to a cover of a rational curve with exactly two components.

Such an admissible cover has by definition a single triple ramification point over a branch point $p_{1}$ lying on $\mathbb{P}_{1}$ or on $\mathbb{P}_{2}$ and not on their intersection. In order to map to $[C]$, it should contain over $\mathbb{P}_{2}$ the curve $C^{\prime}$ and over $\mathbb{P}_{1}$ a rational component $R$ intersecting $C^{\prime}$ exactly at the points $p, q$ and other rational components $R_{j}$, each of which intersects $C^{\prime}$ at a unique point $q_{j}$. Since the gonality of the generic curve of genus $2 k-1$ is $k+1$ (i.e., the minimum degree of a non-constant map of $C^{\prime}$ to $\mathbb{P}_{2}$ ), there is no room for other rational components over $\mathbb{P}_{2}$.

We distinguish two cases: (i) $p_{1} \in \mathbb{P}_{2}$; (ii) $p_{1} \in \mathbb{P}_{1}$. In the first case, if $p_{1} \in \mathbb{P}_{2}$ then the map $C^{\prime} \rightarrow \mathbb{P}_{2}$ is of type (3) and by the choice of $p$ and $q$ it coincides with our $\gamma$. Then we find that $R \rightarrow \mathbb{P}_{1}$ is a $2: 1$ covering and the remaining components $R_{j}$ map isomorphically to $\mathbb{P}_{1}$. We thus retrieve the cover $X$ described in the first paragraph of our proof.

In the second case, if $p_{1} \in \mathbb{P}_{1}$ then $C^{\prime} \rightarrow \mathbb{P}_{2}$ is described by a degree $k+1$ pencil $\gamma^{\prime}$. Then either $R$ or one of the $R_{j}$ contains a ramification point of degree 3 lying over $p_{1}$. If this ramification point lies on $R$ then $\gamma^{\prime}$ has the property that $\gamma^{\prime} \geq 2 p+q$ or $\gamma^{\prime} \geq p+2 q$ which is excluded by lemma 7.2. If some $R_{j}$ contains this ramification point then $q_{j}$ has ramification degree $\geq 3$ which contradicts the generality of $(p, q)$.

We now do the $E_{2}$ case which is similar. For this we need the fact that for a general curve $C^{\prime}$ of genus $2 k-1$ the number of pencils on $C^{\prime}$ of degree $k+1$ and type $(2,2)$ equals

$$
d(k)=12 \frac{(k-1)(k-2)}{k}\left(\begin{array}{c}
2 k \\
k+1
\end{array}\right)
$$

This can be calculated as in Harris [14]. We also need an analogue of lemma 7.2.

LEMma 7.4. Let $C^{\prime}$ be a general curve of genus $2 k-1$ and $p$ a general point of $C^{\prime}$. Then there exists a point $q$ on $C^{\prime}$ such that

1. there exists a unique pencil $\delta$ on $C^{\prime}$ of degree $k+1$ and type $(2,2)$ with $\delta \geq p+q$;

2. there does not exist a pair $\left(\delta^{\prime}, q^{\prime}\right)$ with $\delta^{\prime}$ a pencil of degree $k+1$ and a point $q^{\prime}$ on $C^{\prime}$ with $\delta^{\prime} \geq p+q+2 q^{\prime}$.

Proof. Let $\delta_{j}$ with $j=1, \ldots, d(k)$ be the type $(2,2)$ pencils of degree $k+1$ and let $T_{2}=\cup_{j=1}^{d(k)} T_{\delta_{j}}$ be the union of the trace curves. We now choose a pair $(p, q) \in T_{2}$ which is sufficiently general, i.e., $p$ is not contained in the image under the first projection $\tau_{2}: T_{2} \rightarrow C^{\prime}$ of any multiple point of $T_{2}$ and $T_{3}$ (as defined in lemma 7.2) and $p$ is not contained in any fibre of a $\gamma_{i}$ (as in lemma 7.2) or a $\delta_{j}$ containing a ramification point; this gives $\# \tau_{2}^{-1}(p)=k d(k)$.

We now set $S_{p}=\tau_{2}^{-1}(p)$. We let $\left(\delta_{j}^{\prime}, q_{j}^{\prime}\right)$ for $j=1, \ldots, c(k)$ be the pairs of pencils $\delta_{j}^{\prime}$ of degree $k+1$ and points $q_{j}^{\prime}$ on $C^{\prime}$ with $\delta_{j}^{\prime} \geq p+2 q_{j}^{\prime}$. Now we define

$$
S_{p}^{\prime}=\left\{q^{\prime} \in C^{\prime}: \text { there exists a } j \text { such that } \delta_{j}^{\prime} \geq p+2 q_{j}^{\prime}+q^{\prime}\right\} .
$$


We have $\# S_{p}^{\prime}=(k-2) c(k)$ and we see using the shape of $c(k)$ and $d(k)$ that $\# S_{p}>$ $\# S_{p}^{\prime}$. We can now choose a point $q$ in $S_{p}-S_{p}^{\prime}$ and the unique $\delta_{j}$ with $1 \leq j \leq d(k)$ such that $(p, q) \in T_{\delta_{j}}$. This finishes the proof of the lemma.

We take a generic curve $C^{\prime}$ of genus $2 k-1$ with a pencil $\delta$ of degree $k+1$ and type $(2,2)$ and a couple $(p, q)$ of points as in lemma 7.4. We get a nodal curve $C=C^{\prime} /(p \sim q)$ and a point $[C]$ on the boundary $\Delta_{0}$ of $\overline{\mathcal{M}}_{g}$.

Proposition 7.5. The set-theoretic fibre of the map $\pi^{\prime}: \bar{H}_{k+1,6 k-2,2} / \mathbb{S}_{6 k-2} \rightarrow$ $D_{2}$ over the point $[C]$ consists of one point.

Proof. The decription of the admissible cover representing the unique point is completely similar to the $D_{3}$ case. We analyze again the uniqueness. As before a point in the fibre corresponds to a cover of a rational curve with two irreducible components $\mathbb{P}_{1}$ and $\mathbb{P}_{2}$. Over $\mathbb{P}_{2}$ we have a cover $C^{\prime} \rightarrow \mathbb{P}_{2}$ and over $\mathbb{P}_{1}$ a cover $R \rightarrow \mathbb{P}_{1}$ and a number of smooth rational curves $R_{j}$ mapping with finite degree to $\mathbb{P}_{1}$.

Let $p_{1}$ be the point over which the ramification of type $(2,2)$ occurs. If $p_{1} \in \mathbb{P}_{2}$ we find as above that the admissible cover is the one we want. If $p_{1} \in \mathbb{P}_{1}$ then let $r_{1}, r_{2}$ be the ramification points of type $(2,2)$ over $p_{1}$. We have the following cases:

1. $r_{1}, r_{2} \in R$;

2. $r_{1} \in R, r_{2} \in R_{j}$ for some $j$;

3. $r_{1} \in R_{j_{1}}$ and $r_{2} \in R_{j_{2}}$ for some $j_{1} \neq j_{2}$;

4. $r_{1}, r_{2} \in R_{j}$ for some $j$.

In case 1 ) we conclude that $R \rightarrow \mathbb{P}_{1}$ has degree $\geq 4$, hence the sum of the ramification degrees at $p$ and $q$ is at least 4 , contradicting the generality of $p$ and $q$. In case 2) the degree $R_{j} \rightarrow \mathbb{P}_{1}$ is at least 2 , hence the ramification degree at $q_{j}$ with $q_{j}=R_{j} \cap C^{\prime}$ is at least 2 , contradicting lemma 7.4. The other cases are easy because in case 3 ) the ramification degrees of $q_{j_{1}}$ and $q_{j_{2}}$ are at least 2, contradicting the choice of $(p, q)$, while in case 4$)$ the ramification degree at $q_{j}$ is at least 4 which contradicts the generality of $C^{\prime}$. Thus we are done in all cases.

In order to prove the Theorem we have to analyze the multiplicity.

Our local analysis of the map $\pi: \bar{H}_{k+1,6 k-2,3} \rightarrow D_{3} \subset \overline{\mathcal{M}}_{g}$ over the point $[C]$ is similar to the one described in [16], pages 76-78 for the case of admissible covers with simple branching only. For a similar description over Hurwitz schemes of other types, see [2], Section 3 and [3] p. 46.

We take a point $x$ in the fiber of the covering $\pi: \bar{H}_{k+1,6 k-2,3} \rightarrow D_{3}$ over $[C]$. As we have seen, $x$ corresponds to a covering of the form $X$ defined above - modulo renumbering of the marked simple branch points - and it is a smooth point of the space $\bar{H}_{k+1,6 k-2,3}$. By the uniqueness we proved above, in a neighborhood of the point $[C]$ the variety $D_{3}$ is the image via the map $\pi$ of a neighborhood of the point $x$. We choose a marking of all branch points by marking points $p_{2}, p_{3}$ on $\mathbb{P}_{1}$. If $\sigma$ is the permutation of $\mathbb{S}_{2 k-2}$ interchanging $p_{2}$ and $p_{3}$, then $\sigma x=x$, cf. Remark 4.2. The fixed locus of the permutation $\sigma$ in the neighborhood of $x$ is a divisor $\Delta$. The complement of $\Delta$ in the neighborhood of $x$ corresponds to coverings of smooth curves. Therefore, locally at $[C]$, the image of $\Delta$ corresponds to the intersection of $D_{3}$ with the boundary divisor $\Delta_{0}$ of $\overline{\mathcal{M}}_{g}$. The map $\tau: \bar{H}_{k+1,6 k-2,3} \rightarrow \bar{H}_{k+1,6 k-2,3} /\langle\sigma\rangle$ is locally around $x^{\prime}=\tau(x)$ a degree 2 covering with ramification locus $\Delta$, see [16], bottom of p. 76 .

As is shown in [16], p. 77, the induced map $\lambda: \bar{H}_{k+1,6 k-2,3} /\langle\sigma\rangle \rightarrow D_{3} \subset \overline{\mathcal{M}}_{g}$, has the property that $\lambda^{*}\left(\Delta_{0}\right)=\tau(\Delta)$ with multiplicity one. This implies that $D_{3}$ and $\Delta_{0}$ meet transversally in the neighborhood of $[C]$. Since $[C]$ is locally a generic point of the intersection of $D_{3}$ with $\Delta_{0}$, we conclude that it is a smooth point of $D_{3}$. 
Moreover, since $\lambda^{*}\left(\Delta_{0}\right)=\tau(\Delta)$ with multiplicity one, we find that the ramification index of $x^{\prime}$, which is a generic point, equals 1 . Hence the ramification index at the point $x$ of the map $\pi: \bar{H}_{k+1,6 k-2,3} \rightarrow D_{3} \subset \overline{\mathcal{M}}_{g}$ is 2 and this finishes the proof of the Theorem for the case of $E_{3}$. The analysis for the $E_{2}$ case is similar.

8. The calculation of the class. We shall now carry out the calculation of the class of $D_{2}$. We use the calculation of the class of $D_{3}$ due to Harris in [14], p. 466 and the formula of Kokotov, Korotkin and Zograf in [18]. Harris gives the class of $D_{3}$ (for $k \geq 2)$ as

$$
\left[D_{3}\right]=12 \frac{(2 k-3) !}{(k+1) !(k-2) !}\left[\left(12 k^{2}+46 k-8\right) \lambda-b_{0} \delta_{0}-\sum_{j=1}^{k} b_{j} \delta_{j}\right]
$$

with $b_{0}=2 k^{2}+4 k-1$ and for $b_{j}=2 j(2 k-j)(3 k+2)$ for $j>0$. We can rewrite this as

$$
\left[D_{3}\right]=\frac{3}{2 k-1} N\left[2(k+4)(6 k-1) \lambda-b_{0} \delta_{0}-\sum_{j=1}^{k} b_{j} \delta_{j}\right]
$$

where $N=\left(\begin{array}{c}2 k \\ k+1\end{array}\right) / k=\left(\begin{array}{c}2 k \\ k\end{array}\right) /(k+1)$.

In their paper [18] Kokotov, Korotkin and Zograf give a formula for the (first Chern) class $\lambda_{H}$ of the Hodge bundle on $\bar{H}_{g, d}$ (which is the pull back of the class $\lambda$ of the Hodge bundle on $\overline{\mathcal{M}}_{g}$ ). In our case their formula (Thm. 3, formula (3.13), see also [11], where there is an unfortunate omission of a summation symbol for the index $\mu$ ) reads

$$
\lambda_{H}=\sum_{b_{2}}^{3 k} \sum_{\mu} m(\mu)\left[\frac{b_{2}\left(6 k-b_{2}\right)}{8(6 k-1)}-\frac{1}{12}\left(k+1-\sum_{i} \frac{1}{m_{i}}\right)\right] \delta_{\mu}^{\left(b_{2}\right)},
$$

where $b_{2}$ is the number of marked point on $\mathbb{P}_{2}, \mu=\left(m_{i}\right)$ 's are the ramifications over $s, \delta_{\mu}^{\left(b_{2}\right)}$ the corresponding boundary divisor and $m(\mu)$ is the least common multiple of the $m_{i}$ 's; cf. the proof of Thm. 3 of loc. cit. Here we work on the stack, cf. [11].

We apply the push forward $\pi_{*}$ to this formula and plug in the result of Harris. For $E_{0}$ we have $k+1$ points over $s$ of ramification degree $m_{i}=1$, hence $m(\mu)=1$. For $E_{2}$ we have $k-3$ points of ramification degree 1 and two of ramification degree 2 , so $m(\mu)=2$. Similarly, for $E_{3}$ we have $k-2$ points of ramification degree 1 and one of ramification degree 3 , so $m(\mu)=3$. For $E_{j, c}$ we have $k-j+2 c$ points over $s$ with ramifications degree 1 and one of ramification degree $j+1-2 c$, so $m(\mu)=j+1-2 c$. This yields:

Proposition 8.1. $\pi_{*}\left(\lambda_{H}\right)$ of the Hodge class $\lambda_{H}$ is given by

$$
\begin{aligned}
& \frac{(3 k-1)}{2(6 k-1)} \pi_{*}\left[E_{0}\right]-\frac{1}{2(6 k-1)} \pi_{*}\left[E_{2}\right]+\frac{3 k-5}{6(6 k-1)} \pi_{*}\left[E_{3}\right]+ \\
& \sum_{j=1}^{k} \sum_{c=0}^{[j / 2]}(j+1-2 c)\left[\frac{(6 k-3 j)(3 j)}{8(6 k-1)}-\frac{1}{12}\left(j+1-2 c-\frac{1}{j+1-2 c}\right)\right] \pi_{*}\left[E_{j}, c\right] .
\end{aligned}
$$

Here we have to interpret the classes $\pi_{*}\left[E_{0}\right], \ldots, \pi_{*}\left[E_{j, c}\right]$ in the right way since we are working on the stack $\overline{\mathcal{M}}_{g}$. By applying $\pi_{*}$ with its degree $\operatorname{deg}(\pi)=(6 k) ! N$ 
and using Proposition 3.3 and Theorem 7.1 we find

$$
\pi_{*}\left(\lambda_{H}\right)=\operatorname{deg}(\pi) \lambda_{\mathcal{M}}, \quad \pi_{*}\left[E_{0}\right]=\frac{\operatorname{deg}(\pi)}{2} \delta_{0} .
$$

Indeed, a generic admissible cover of $E_{0}$ admits no non-trivial automorphisms fixing the marked points. (That the degree of $\pi$ restricted to $E_{0}$ is $\operatorname{deg}(\pi) / 2$ is due to the fact that such an admissible cover allows an involution that does not fix the marked points.) Similarly, we find $\pi_{*}\left[E_{3}\right]=\frac{(6 k) !}{2}\left[D_{3}\right]$, with the class of $D_{3}$ given above. Along $E_{2}$ an admissible cover has a $\mathbb{Z} / 2 \mathbb{Z} \times \mathbb{Z} / 2 \mathbb{Z}$ in its automorphism group preserving the marked points with the two generators corresponding to the covering involutions on $S_{1}$ and $S_{2}$ over $\mathbb{P}_{1}$ (see Prop. 3.1, i). But locally along $E_{2}$ the infinitesimal defomation space has a normal crossing singularity, cf. Lemma 4.3. We go to the normalization and interpret the formula of [18] there in the stack sense (cf. the remarks at the end of $\S(3.1)$ of [18] and the discussion in [11]). Over $E_{2}$ this is a $2: 1$ cover. So taking into account these factors $2 / 2^{2}$ of 2 we find $\pi_{*}\left[E_{2}\right]=(6 k) !\left[D_{2}\right] / 2$.

From Proposition 3.3 we get for $j>0$

$$
\pi_{*}\left[E_{j, c}\right]=\frac{(6 k) !(j+1-2 c)^{2}}{(j+1)(2 k-j+1)}\left(\begin{array}{c}
j+1 \\
c
\end{array}\right)\left(\begin{array}{c}
2 k-j+1 \\
k+1-c
\end{array}\right) \delta_{j} .
$$

We put for $i \in \mathbb{Z}_{\geq 1}$

$$
A_{i}(j)=\frac{1}{(j+1)(2 k+1-j)} \sum_{c=0}^{[j / 2]}(j+1-2 c)^{i}\left(\begin{array}{c}
j+1 \\
c
\end{array}\right)\left(\begin{array}{c}
2 k-j+1 \\
k+1-c
\end{array}\right) .
$$

Then with $N=\left(\begin{array}{c}2 k \\ k+1\end{array}\right) / k$ we have

$$
A_{2}(j)=N, \quad A_{4}(j)=\left(1+\frac{3 j(2 k-j)}{2 k-1}\right) N,
$$

while for $A_{3}(j)$ we get if $j$ is even

$$
A_{3}(j)=\frac{j(2 k-j)+k}{k(k+1)}\left(\begin{array}{c}
j \\
{[j / 2]}
\end{array}\right)\left(\begin{array}{c}
2 k-j \\
k-[j / 2]
\end{array}\right)
$$

and for $j$ odd

$$
A_{3}(j)=\frac{(j+1)(2 k-j)}{k(k+1)}\left(\begin{array}{c}
j+1 \\
1+[j / 2]
\end{array}\right)\left(\begin{array}{c}
2 k-j-1 \\
k-1-[j / 2]
\end{array}\right) .
$$

By multiplying by $2(6 k-1)$ and bringing $\pi_{*}\left[E_{2}\right]$ to the other side in the equation for $\pi_{*}\left(\lambda_{H}\right)$ in Proposition 8.1 we get

$$
\begin{aligned}
\pi_{*}\left[E_{2}\right]= & -2(6 k-1) \pi_{*}\left(\lambda_{H}\right)+\frac{3 k-5}{3} \pi_{*}\left[E_{3}\right]+(3 k-1) \pi_{*}\left[E_{0}\right]+ \\
& (6 k) ! \sum_{j=1}^{k}\left[\frac{(6 k-3 j)(3 j)}{4} A_{3}(j)+\frac{6 k-1}{6}\left(-A_{4}(j)+A_{2}(j)\right)\right] \delta_{j} .
\end{aligned}
$$

Dividing by $(6 k)$ ! we find

$$
\begin{aligned}
{\left[D_{2}\right] / 2=} & -2(6 k-1) N \lambda_{\mathcal{M}}+\frac{3 k-5}{6}\left[D_{3}\right]+\frac{3 k-1}{2} N \delta_{0}+ \\
& \sum_{j=1}^{k}\left[\frac{(6 k-3 j)(3 j)}{4} A_{3}(j)+\frac{6 k-1}{6}\left(-A_{4}(j)+A_{2}(j)\right)\right] \delta_{j} .
\end{aligned}
$$


Only the first two terms on the right hand side contribute to the coefficient of $\lambda_{\mathcal{M}}$ and the contribution is

$$
\begin{array}{r}
-2(6 k-1) N \lambda_{\mathcal{M}}+\frac{3 k-5}{6} \frac{3}{2 k-1} N 2(k+4)(6 k-1) \lambda_{\mathcal{M}}= \\
3 N \frac{6 k-1}{2 k-1}(k-2)(k+3) \lambda_{\mathcal{M}} .
\end{array}
$$

The coefficient of $\delta_{0}$ comes from the second and third term on the right hand side. It is

$$
-\frac{3 k-5}{6} \frac{3}{2 k-1} N\left(2 k^{2}+4 k-1\right)+(3 k-1) \frac{N}{2}=-\frac{N}{2 k-1}(k-2)\left(3 k^{2}+4 k-1\right) .
$$

The coefficient of $\delta_{j}, j \geq 1$, comes from the second and fourth term on the right hand side. We get

$$
\begin{array}{r}
-\frac{1}{2} N \frac{3 k-5}{2 k-1} 2 j(2 k-j)(3 k+2)+\frac{9(2 k-j) j}{4} A_{3}(j)-N \frac{(6 k-1) j(2 k-j)}{2(2 k-1)}= \\
-\frac{3 N j(2 k-j)}{2(2 k-1)}\left(6 k^{2}-4 k-7\right)+\frac{9}{4} j(2 k-j) A_{3}(j) .
\end{array}
$$

This concludes the proof of the theorem.

9. A final check. Our main result gives zero for $k=2$, as it should. It also satisfies the relation $c_{\lambda}+12 c_{0}-c_{1}=0$ given in Lemma 3.1 of [14]. But these checks using homogeneous linear relations leave the possibility of common factor in the coefficients $c_{\lambda}, c_{0}, \ldots, c_{k}$. To rule this out we consider a test curve in $\overline{\mathcal{M}}_{g}$. Take a general curve $B$ of genus $g-1$, a general point $p \in B$ and identify in the blow-up of $B \times B$ at $(p, p)$ the diagonal with the section $\{p\} \times B$. This gives a family $\pi: S \rightarrow B$ of one-nodal curves with

$$
B \cdot \lambda=0, \quad B \cdot \delta_{0}=2-2 g, \quad B \cdot \delta_{1}=1, \quad \text { and } B \cdot \delta_{j}=0 \text { for } j \geq 2 .
$$

Lemma 9.1. We have $B \cdot D_{2}=(k-1)(k-2)(12 k+10) N$.

Proof. We have $B \cdot D_{2}=S_{p}+2 S_{p}^{\prime}$ with $S_{p}$ and $S_{p}^{\prime}$ defined in the proof of Lemma 7.4. The argument is similar to that of [14], Lemma 3.9. Set-theoretically we have $D_{2} \cdot B=S_{p} \cup S_{p}^{\prime}$. The argument for the multiplicity of $S_{p}$ is similar to that of loc. cit. As to the multiplicity of $S_{p}^{\prime}$, an analysis shows that it equals 2 due to the involution involved here. In the proof of Lemma 7.4 we gave the cardinalities: $\# S_{p}=k d(k)$ and $\# S_{p}^{\prime}=(k-2) c(k)$. This proves the Lemma.

On the other hand using the intersection numbers of $B$ with $\lambda$ and the $\delta_{i}$ we get by our Theorem

$$
B \cdot D_{2}=-2(2 k-1) c_{0}+c_{1}=2(k-1)(k-2)(6 k+5) N,
$$

in perfect agreement with the Lemma 9.1.

As a second type of check, one can use the calculations above to calculate the class of the ramification divisor $D$ on the universal curve.

Recall that the Picard group of the universal curve is generated by classes $\lambda, \omega$, $\delta_{0}^{\prime}$, and $\delta_{i}^{\prime}$ for $i=1, \ldots, g-1$. With $\pi: \overline{\mathcal{C}}_{g} \rightarrow \overline{\mathcal{M}}_{g}$ the natural map we have $\pi^{*}\left(\delta_{0}\right)=\delta_{0}^{\prime}$ 
and $\pi^{*}\left(\delta_{i}\right)=\delta_{i}^{\prime}+\delta_{g-i}^{\prime}$ fir $i=1, \ldots, g-1$. Since the generic curve of genus $g=2 k$ carries finitely many $g_{k+1}^{1}$ 's we can consider the divisor $D$ on $\overline{\mathcal{C}}_{g}$ that is the closure of the locus of all ramification points of the $g_{k+1}^{1}$ 's on the general curve $C$. The generic degree of the restriction of $\pi$ to $D$ is $6 k N$. The calculation of the class of $D$ reduces to a calculation of

$$
\pi_{*}(D \omega)=\frac{1}{(6 k) !} p_{*}\left(\frac{1}{2} q^{*} \psi-2 E_{2}-2 E_{3}\right) .
$$

One finds the following class.

Proposition 9.2. The class of $D$ in the Picard group of $\overline{\mathcal{C}}_{g}$ is

$$
[D]=a \omega+\frac{a}{6 k}\left(6(k+2) \lambda-(k+1) \delta_{0}^{\prime}\right)-\ldots
$$

where $a=\frac{3 k N}{2 k-1}$ and $\ldots$ refers to an expression $\sum_{i=1}^{g-1} c_{i} \delta_{i}^{\prime}$.

Note that the expression $(a / 6 k)\left(6(k+2) \lambda-(k+1) \delta_{0}\right)-\ldots$ is exactly the expression for the class of the $(k+1)$-gonal locus in $\overline{\mathcal{M}}_{2 k+1}$. The class of $D$ has been calculated by Logan and by Farkas, [9], Thm. 4.9, and our result agrees with this.

\section{REFERENCES}

[1] E. Arbarello, M. Cornalba, Ph. Griffiths, and J. Harris, Geometry of Algebraic Curves I, Grundlehren der mathematischen Wissenschaften 267, 1985, Springer Verlag.

[2] F. Cukierman, Families of Weierstrass points, Duke Math. J., 58 (1989), pp. 317-346.

[3] S. Diaz, Exceptional Weierstrass points and the divisor on moduli space they define, Mem. Amer. Math. Soc., 56, 1985.

[4] S. DiAz, Tangent spaces in moduli, via deformations with applications to Weierstrass points, Duke Math. J., 51 (1984), pp. 905-922.

[5] D. Eisenbud And J. Harris, Limit linear series, Basic theory, Inventiones Mathematicae, 85 (1986), pp. 337-371.

[6] D. EISENBUD AND J. HARRIS, Irreducibility of some families of linear series with Brill-Noether number -1, Ann. Scient. Ec. Norm. Sup., 22 (1989), pp. 33-53.

[7] C. FABer And R. PAndharipande, Relative maps and tautological classes, Journal of the EMS, 7 (2005), pp. 13-49.

[8] G. Farkas, The Fermat cubic and special Hurwitz loci in $\overline{\mathcal{M}}_{g}$, Bull. Belg. Math. Soc. - Simon Stevin, 16:5 (2009), pp. 831-851.

[9] G. Farkas, Koszul divisors on moduli spaces of curves, American J. of Math., 131 (2009), pp. 819-867.

[10] W. Fulton, Hurwitz schemes and irreducibility of moduli of algebraic curves, The Annals of Mathematics, 90 (1969), pp. 542-575.

[11] G. VAn Der Geer and A. Kouvidakis, The Hodge bundle on Hurwitz spaces, Pure and Applied Mathematics Quarterly, 7 (2011), pp. 1297-1307.

[12] G. VAN DER GeER AND A. KouvidAKIS, Rational correspondences between moduli spaces of curves defined by Hurwitz spaces, J. Pure and Applied Algebra (2011), doi 10.1016/j.jpaa.2011.10.017.

[13] Ph. GRIffiths And J. HARRIS, On the variety of special linear systems on a general algebraic curve, Duke Math. J., 47 (1980), pp. 233-272.

[14] J. HARris, On the Kodaira dimension of the moduli space of curves II. The even-genus case, Inventiones Mathematicae, 75 (1984), pp. 437-466.

[15] J. Harris And I. Morrison, Moduli of Curves, Graduate Texts in Mathematics, 187, 1998, Springer Verlag.

[16] J. Harris And D. Mumford, On the Kodaira dimension of the moduli space of curves, Inventiones Mathematicae, 67 (1982), pp. 23-86.

[17] P. Kluitman, Hurwitz action and finite quotients of braid groups, Contemporary Mathematics, 78 (1988), pp. 299-325.

[18] A. Kokotov, D. Korotkin, And P. Zograf, Isomonodromic tau function on the space of admissible covers, Adv. Math., 227 (2011), pp. 586-600. 
G. VAN DER GEER AND A. KOUVIDAKIS 